Año LXXXII. urtea $279-2021$

Enero-abril Urtarrila-apirila

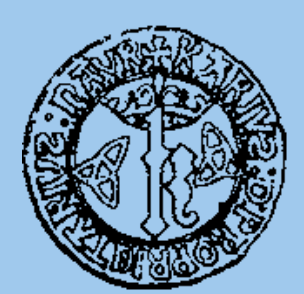

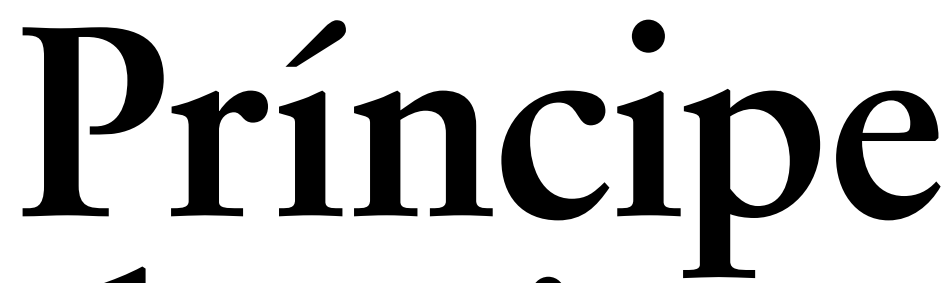

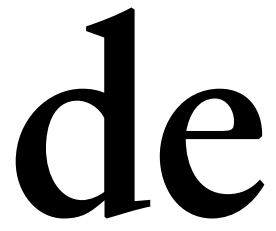

SEPARATA

Donación del fondo bibliográfico y documental Gustav Henningsen y Marisa Rey a la Universidad Pública de Navarra

Iñaki MONTOYA ORTIGOSA 


\section{Sumario / Aurkibidea}

\section{Príncipe de Viana}

Año LXXXII • n. ${ }^{\circ} 279$ - enero-abril de 2021

LXXXII. urtea $\cdot 279$. zk. 2021 ko urtarrila-apirila

\section{GUSTAV HENNINGSEN / MARISA REY-HENNINGSEN}

\section{Homenaje / Omenaldia}

Ignacio Panizo (coord./koord.)

Vol. II. lib.

\section{ESTUDIOS DE INVESTIGACIÓN EN HOMENAJE \\ A GUSTAV HENNINGSEN Y MARISA REY-HENNINGSEN / \\ IKERKETA LANAK, GUSTAV HENNINGSENEN \\ ETA MARISA REY-HENNINGSENEN OMENEZKOAK}

Entre impostores anda el juego en el Siglo de Oro. Dos vizcaínos ante la Inquisición y unos conversos toledanos próximos a Garibay

Un formulario de la Secretaría de Aragón del Consejo de la Inquisición (BC, ms. 2168)

Anna Gudayol

Las cartillas editadas en Estella e incautadas en Medellín por la Inquisición (1561)

Javier Itúrbide Díaz

El Tribunal de Navarra y sus funcionarios inquisitoriales:

algunas observaciones

Consuelo Juanto Jiménez

Goya investigado por la Inquisición: la censura de los Caprichos en 1804

$\mathrm{El}$ «Informe del inquisidor Avellaneda» sobre las complicidades brujeriles en los valles centrales y orientales del Pirineo navarro José M. Floristán Imízcoz, Jesús Moya Mangas 


\section{Sumario / Aurkibidea}

La Inquisición en Tudela durante los siglos XVI y XVII:

presencia e incidencia social

Iñigo Pérez Ochoa

La huella de la Inquisición en la Biblioteca de Navarra

Roberto San Martín Casi

Los memoriales como fuente para el estudio de la Inquisición

en el Tribunal de Navarra (1609-1621)

Bárbara Santiago Medina

De comisarios, confesores y vecinos: la proyección de la Inquisición

a finales del Antiguo Régimen

Marina Torres Arce

Los niños como víctimas y los niños como verdugos en la caza de brujas:

Navarra, siglo XVI

Jesús M. Usunáriz

Volver a las relaciones de causas. El ejemplo de los berberiscos

del reino de Granada

Bernard Vincent

\section{LOS TRABAJOS Y LOS DÍAS DEL AÑO 2020 / 2020ko LANAK ETA EGUNAK}

Tesis doctorales sobre temática navarra de ciencias humanas, sociales y jurídicas, leídas en 2020

(Según la Base de datos Teseo del Ministerio de Educación)

Narrativa de autores navarros en castellano, año 2020

Mikel Zuza Viniegra

Euskarazko literaturak 2020an zer?

Ángel Erro Jiménez

Donación del fondo bibliográfico y documental Gustav Henningsen y Marisa

Rey a la Universidad Pública de Navarra

Iñaki Montoya Ortigosa

Formación en Archivística y Gestión Documental en la Universidad de Navarra

Yolanda Cagigas Ocejo

6 Príncipe de Viana (PV), 279, urtarrila-apirila, 2021

ISSN: 0032-8472 | ISSN-e: 2530-5824 | ISSN-L: 0032-8472

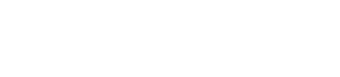




\section{Sumario / Aurkibidea}

Apuntes sobre etnografía, folclore y cultura tradicional David Mariezkurrena Iturmendi

El alivio y reparo. La forma audiovisual

Marga Gutiérrez Díez

Proceso de decantación. Exponer en 2020

Mireya Martín Larumbe

Exposición «Maleficium. Navarra y la caza de brujas. Siglos XIV-XVII»

(Archivo Real y General de Navarra, julio-diciembre, 2020)

Jesús M. Usunáriz

Carlos Cánovas

Alicia Ezker Calvo

Discurso Premio Príncipe de Viana 2020

Carlos Cánovas Ciaurriz

Currículums

Analytic Summary

Normas para la presentación de originales / Idazlanak aurkezteko arauak /

Rules for the submission of originals 


\title{
Donación del fondo bibliográfico y documental Gustav Henningsen y Marisa Rey a la Universidad Pública de Navarra
}

\author{
Iñaki Montoya Ortigosa \\ Jefe de Sección de Gestión de Documentos y Archivo General \\ Universidad Pública de Navarra \\ Agirien Kudeaketa eta Artxibo Nagusiko Ataleko burua \\ Nafarroako Unibertsitate Publikoa \\ inaki.montoya@unavarra.es
}

DOI: https://doi.org/10.35462/pv.279.16

El prestigioso matrimonio de investigadores Gustav Henningsen y Marisa Rey ha generado, a lo largo de su carrera profesional, un conjunto de materiales de investigación, así como de diversa documentación de archivo y una biblioteca particular de gran valor científico. Este fondo documental y bibliográfico está integrado por los documentos y libros generados, recibidos o recopilados para la realización de sus destacados proyectos de investigación, especialmente aquellos relacionados con el estudio de la brujería y la Inquisición española y que han tenido notoria repercusión en la comunidad científica internacional. También contiene parte de su archivo privado personal, que ayuda a comprender y a contextualizar dichas investigaciones.

Gustav Henningsen y Marisa Rey fueron muy cuidadosos, en la gestión de este conjunto de documentación, organizando y clasificando sus documentos en carpetas, portafolios, sobres, clasificadores, cajas de archivo, etc.

Tras su jubilación, el matrimonio se instaló en la localidad malagueña de Pizarra, en una espaciosa casa de su propiedad. Allí trasladaron todo su archivo y biblioteca, que distribuyeron en dos amplios despachos y en su garaje.

En el verano de 2019 Gustav Henningsen y Marisa Rey, se trasladaron de forma urgente, por motivos de salud, a su residencia habitual de Copenhague, dejando el fondo documental y bibliográfico en su casa de Pizarra. Siendo conscientes del valor del fondo, el matrimonio solicitó ayuda al historiador pamplonés Ignacio Panizo, archivero del Cuerpo Facultativo de Archiveros del Estado, para que les ayudase a gestionar el destino final de la documentación. El matrimonio mantiene con Ignacio Panizo una estrecha 
relación profesional y de amistad desde los años en los que este era el responsable de la Sección de Inquisición del Archivo Histórico Nacional.

Los Henningsen-Rey, junto con Panizo, establecieron los siguientes objetivos para el destino final del fondo:

- La documentación quedaría en España, preferentemente en una institución pública.

- El archivo, la documentación de investigación y la biblioteca tendrían un destino único, sin dividir en diferentes instituciones.

- Era necesario un tratamiento técnico archivístico y bibliotecario del fondo para darle acceso y uso a investigadores y estudiosos.

Diversas instituciones públicas y privadas europeas y españolas se mostraron interesadas en recibir esos fondos, si bien, tras estudiar las diferentes opciones, el matrimonio Henningsen-Rey optó por entregar el grueso de sus fondos a la Universidad Pública de Navarra/Nafarroako Unibertsitate Publikoa, y una pequeña parte al Museo del Pueblo Gallego/Museo do Pobo Galego y a la Biblioteca Nacional.

Los trámites para la donación a la Universidad Pública de Navarra fueron emprendidos por el secretario general, Roldán Jimeno, a principios de marzo de 2020. El inmediato confinamiento por la pandemia de la COVID-19 impidió viajar a Málaga para examinar los materiales, lo que no se pudo hacer hasta finales de junio. El análisis de estos fondos albergados en Pizarra, que desarrollamos como responsables del Archivo de la UPNA, partió de un detallado informe previo realizado por Ignacio Panizo tras una visita a finales de enero de 2020. Por diversas circunstancias, la documentación se había desorganizado y buena parte de su biblioteca se había trasladado a Copenhague y Griñón (Madrid). Parte de los fondos, además, habían sido dañados por una inundación de los bajos del garaje, que afectó a alguno de los materiales depositados en las estanterías más bajas. Para evitar males mayores, un familiar tuvo que distribuir el material de las baldas inferiores a diferentes huecos de las baldas más elevadas.

Todas las partes implicadas considerábamos que la documentación tenía un altísimo riesgo de pérdida o deterioro al encontrarse en una casa deshabitada. Además, a los Henningsen les urgía poner a la venta la casa de Pizarra, pero antes, lógicamente, era necesario sacar la documentación de la casa. Era urgente, por lo tanto, trasladar la documentación a un lugar seguro.

Por los motivos expuestos, cuando terminó el primer estado de alarma, el 21 de junio de 2021, la UPNA propuso trasladar de urgencia la documentación al Archivo y Biblioteca de la UPNA. Una vez en la UPNA, se haría el examen y valoración de los fondos, al objeto de aceptar o no la donación. Se consideraba, además, que realizar dos viajes, uno para realizar la valoración y otro para proceder a organizar el traslado, era sumamente arriesgado, ya que entre un viaje y otro era posible que se decretase un nuevo estado de alarma o que se produjese algún deterioro irreversible de los documentos. El cambio de estrategia fue aceptado por los Henningsen. 
La mudanza de los documentos y libros de Pizarra se llevó a cabo entre el 27 de junio y el 1 de julio de 2020. A la vista de las condiciones en que se encontraba la documentación en Pizarra, habituales en inmuebles deshabitados (humedad, falta de ventilación, presencia de algún insecto, etc.), cabe decir que la actuación fue totalmente necesaria y adecuada para proteger este valioso fondo. La mudanza fue preparada, controlada y dirigida en origen por el que suscribe, con la ayuda y coordinación telefónica de Ignacio Panizo, especialmente en lo referente a documentos, fotografías y algunos efectos personales de los Henningsen, que no eran objeto de traslado. Finalmente, el 1 de julio el fondo documental y bibliográfico ingresó en las instalaciones de la UPNA; el fondo bibliográfico en su Biblioteca General y el fondo documental en su Archivo General.

El resto de fondos bibliográficos ingresaron en la Biblioteca de la UPNA a finales de agosto de 2020 (Copenhague) y a mediados de febrero de 2021 (Griñón).

La valoración del fondo documental y bibliográfico, realizada en sendos informes de la Biblioteca General y del Archivo General fue, como cabía esperar, positiva.

El principal interés del fondo bibliográfico reside en que contiene los títulos y ejemplares que han sustentado las investigaciones de Gustav Henningsen y Marisa Rey y complementan y realzan el valor de su archivo. Destacan entre ellos un centenar de ejemplares publicados entre mediados del siglo XVIII y principios del siglo XX, considerados de fondo antiguo, de valor histórico y documental.

En lo referente al fondo documental, es de especial interés histórico y científico, al tratarse, principalmente, de las fuentes primarias sobre las que Gustav Henningsen basó su obra intelectual y científica. La obra del investigador danés tiene proyección internacional y una indudable repercusión en la comunidad científica. Supuso un gran avance en los estudios de la Inquisición y aportó una indudable mejora en la historiografía sobre la brujería, estableciendo además la metodología para su estudio. Una parte fundamental de su obra investigadora se centra en los extraordinarios y extensos informes del inquisidor Alonso de Salazar y Frías sobre la gran persecución de la brujería vasca, especialmente los referidos a Zugarramurdi y toda la Montaña de Navarra (1609-1614). A este estudio dedicó su tesis The Witchets' Advocate. Basque Witchcraft and the Spanish Inquisition (1609-1614), publicada en numerosos idiomas, temática en la que fue profundizando, posteriormente, en numerosos artículos de revista, comunicaciones en congresos, etc. Nos hallamos ante el archivo personal de uno de los mayores expertos mundiales en el estudio de la brujería, así como del mayor experto sobre la brujería de la Montaña navarra; un material valiosísimo para cualquier estudioso de la brujería e Inquisición española en general y de la brujería en Navarra en particular.

Por su parte, Marisa Rey, además de colaboradora y traductora de la obra de Gustav Henningsen es antropóloga y folklorista experta en cuentos populares europeos, especialmente gallegos -a los que dedicó su tesis doctoral-, y daneses. 
En el análisis preliminar, se ha podido constatar que el fondo documental está compuesto por:

- Copias de documentos de archivos españoles y europeos que son la base de su investigación. Este material estaba organizado (en gran parte incluso encuadernado) por Gustav Henningsen, pero actualmente ha perdido parte de su organización original. No obstante, debido a la forma minuciosa de trabajo de Henningsen, estimamos que la organización original puede ser reconstruida en gran medida.

Sin ánimo de exhaustividad, dentro de este grupo se ha observado la existencia de los siguientes materiales:

- Copias de documentos de archivos que contienen documentos de la Inquisición o relacionados con sus investigaciones, destacando por su volumen las provenientes del fondo Inquisición del Archivo Histórico Nacional.

- Copias de índices e inventarios de archivos y documentación útiles para la investigación.

- Fichas y recopilaciones bibliográficas.

- Borradores y documentación recopilada para elaboración de sus artículos (artículos de revistas, congresos, etc.). Se deberá valorar su permanencia en el fondo o su expurgo parcial o total.

- Artículos y trabajos de otros autores con notas y análisis marginales.

- Correspondencia profesional como privada tanto de Gustav Henningsen como de Marisa Rey.

- Documentación familiar de los antepasados de Gustav Henningsen. No tiene gran volumen, pero está escrita, lógicamente, en danés.

- Fajos misceláneos de documentos: documentación personal y profesional diversa.

Para poder medir su valor se han realizado muestreos aleatorios de la documentación. No obstante, la verdadera dimensión del valor del fondo documental se apreciará conforme progresen los trabajos de organización y descripción de la documentación. En el análisis preliminar se ha llegado a las siguientes conclusiones:

- La documentación de mayor interés es, a priori, todas las copias de documentos recopiladas por Gustav Henningsen en los diversos archivos españoles y europeos. Aunque la documentación no pueda ser considerada original, sí que puede considerarse original las anotaciones de Gustav Henningsen, su organización, etc. Esto último aporta un gran valor. Es necesario recomponer la organización dada por Henningsen y vincularla con los fondos de origen (AHN, Simancas, ARGN, etc.). Una vez reestablecido y comprendida la organización original, será un material de imprescindible para cualquier investigador que quiera ahondar en el estudio de la brujería e Inquisición. Es posible que este material, una vez organizado, pueda constituir el embrión de un centro de documentación sobre estos temas. Sin embargo, hay que tener en cuenta que Gustav Henningsen obtuvo estas copias con fines de investigación, por lo que, cualquier otro uso de este material por terceros debe tener en cuenta las posibles restricciones de tipo legal, especialmente en lo referente a su publicación y difusión. 
- En cuanto a lo que se puede considerar más estrictamente su archivo personal, en su mayoría se trata de correspondencia, de gran interés para aquellos investigadores que quieran investigar estudiar las figuras de Gustav Henningsen o de Marisa Rey desde el punto de vista profesional.

El Convenio de depósito y cesión de derechos de explotación se firmó en febrero de 2021 por parte del matrimonio Henningsen-Rey y el Rector de la UPNA, Ramón Gonzalo. La UPNA ha asumido los siguientes compromisos y deberes con respecto a los donantes y al propio fondo:

1. Organizar el fondo documental y bibliográfico de acuerdo con criterios técnicos archivísticos y bibliotecarios.

2. Elaborar los instrumentos de descripción pertinentes para poner este material a disposición de los investigadores y del público en general, facilitando su acceso y difusión.

3. A que conforme avancen los trabajos de organización y descripción del fondo, se valorará bajo criterios técnicos, qué documentación es susceptible de digitalización y su puesta a disposición de los investigadores a través del futuro Portal de Archivo de la UPNA.

Los donantes tendrán acceso mediante claves, a través del Portal de Archivo, a todas las descripciones archivísticas del fondo documental que se hayan realizado, así como a todos los documentos que se hayan digitalizado.

4. Mantener el fondo documental y bibliográfico objeto de la donación en las condiciones de seguridad y conservación que garanticen su preservación.

5. Mantener la unidad intelectual del fondo documental y bibliográfico con el objetivo de no devaluar su valor cultural. No obstante, en virtud de su naturaleza como patrimonio documental o bibliográfico, podrá ser tratado técnicamente de acuerdo con dicha naturaleza y ubicado, para su mejor conservación y uso, en el Archivo General, en el caso del fondo documental y en la Biblioteca Universitaria, en el caso del fondo bibliográfico.

6. Convenir la citación en el uso público del fondo documental de la forma siguiente: (C) Archivo Universidad Pública de Navarra / Fondo Gustav Henningsen y Marisa Rey

7. Respetar los derechos morales, respecto a la identificación del autor en cualquier uso que la Universidad Pública de Navarra haga de este material y velar para que en todos los usos públicos por parte de terceros se respeten los derechos morales del autor.

8. Entregar cinco ejemplares, por parte de la UPNA a la familia Henningsen-Rey, de aquellas publicaciones editadas por la propia Universidad que hayan utilizado y mencionado el fondo archivístico Gustav Henningsen y Marisa Rey-Henningsen.

El 18 de marzo de 2021, por resolución rectoral, se aceptó la donación y la cesión de todos los derechos de explotación del este fondo, a la Universidad Pública de Navarra. Así mismo se agradecía esta donación a Gustav Henningsen y Marisa Rey dicha donación «reconociendo el interés histórico, cultural y científico e investigador de este fondo, que tiene tanto para la sociedad en su conjunto, como, en particular, para la 
Universidad Púbica de Navarra, en aras al mejor el cumplimiento de sus fines culturales e investigadores».

El catálogo del fondo bibliográfico donado ya está disponible para su consulta en el OPAC de la Biblioteca de la UPNA https://biblioteca.unavarra.es/abnetopac/abnetcl. cgi/O7163/IDeb49136e/NT18?ACC=257\&DI=7131

Sin duda, la adquisición del fondo Gustav Henningsen y Marisa Rey supone un hito y un reto ineludible para la UPNA en relación a los fondos que componen su patrimonio documental. Un hito porque supone el enriquecimiento del patrimonio documental de la UPNA con un fondo personal imprescindible para cualquier investigación sobre la brujería y la Inquisición española a nivel internacional. Pero, sobre todo, supone un reto ineludible para la UPNA: por el compromiso adquirido con los donantes, por la excepcionalidad del fondo, por la complejidad de su tratamiento archivístico y por el interés que tiene este fondo, tanto en Navarra como a nivel internacional.

Es un reto que la UPNA debe aprovechar y convertir en una oportunidad para el desarrollo de su archivo histórico que, además de dar servicio a la investigación histórica, pueda ser un recurso también para la docencia, especialmente para el recientemente creado Grado de Historia y Patrimonio.

Pero especialmente, el rescate y la futura puesta a disposición de este fondo documental y bibliográfico, es una buena noticia para el desarrollo de la investigación científica en unos temas que todavía siguen interesando y apasionando a la sociedad actual. 